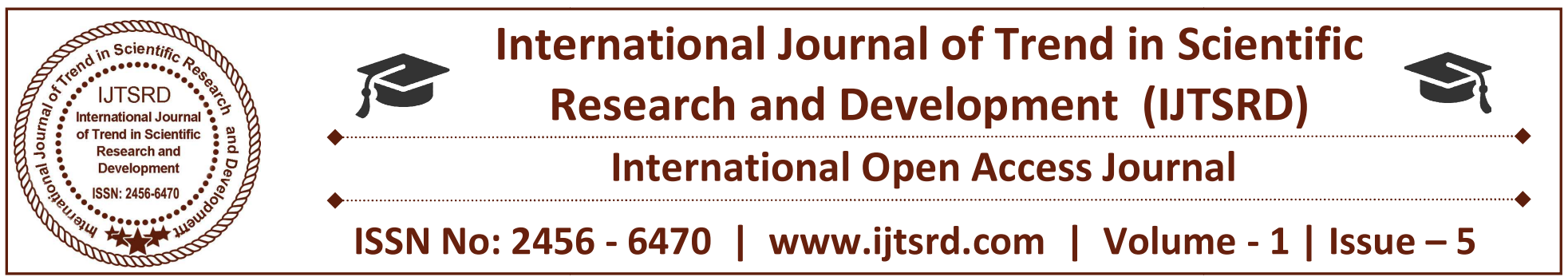

\title{
Performance Analysis of Bayesian methods to for the spectrum utilization in cognitive radio
}

\author{
Abdul Hameed Ansari \\ Pravara Rural Engineering College, Loni
}

\author{
Narode Sweety $S$ \\ Pravara Rural Engineering College, Loni
}

\begin{abstract}
Cognitive radio is an exciting wireless technology that has been introduced for the efficient used of spectrum. Using cognitive radios (CRs), the secondary users (unlicensed users) are allowed to use the spectrum which is originally allocated to primary users (PUs) as far as the active primary users are not using it temporarily. In order to prevent harmful interference to primary users, the SUs need to perform spectrum sensing before transmitting signal over the spectrum. In this paper we use an optimal Bayesian detector for digitally modulated primary user to improve the spectrum utilization, without prior knowledge of transmitted sequence of the primary signals. And further suboptimal detectors in low and high SNR regime. We provide the performance analysis in terms of Detection probability and False alarm probability.
\end{abstract}

Keywords: Cognitive radio, Spectrum sensing, spectrum utilization, Energy Detector, Bayesian Detector

\section{Introduction}

The huge demand for wireless communication raises the need to efficient use of the available spectrum resources. A recent survey made by federal communication commission (FCC) of spectrum utilization has indicated that the actual licensed spectrum is largely unutilized in vast temporal and geographic dimensions. So the Spectrum scarcity is due to the inefficient spectrum management rather than spectrum shortage. To address this problem, cognitive radio has emerged as a desirable technology that has ability to deal with the stringent requirement and scarcity of the radio spectrum and thereby increases the spectral efficiency.

In cognitive radios, the secondary users are allowed to use the spectrum which is originally allocated to primary users as far as primary users are not using it temporarily. It is known as opportunistic spectrum access (OSA). To avoid harmful interference to the primary users, the Secondary unlicensed users have to perform spectrum sensing before transmission over the spectrum. Spectrum sensing is a process in which secondary unlicensed users keep sensing the spectrum to determine whether the PU is transmitting or not. While detecting if PU is absent than the SUs can use those frequencies for transmission. This will help to increase overall spectrum utilization and also in turn increase the spectrum efficiency [6].

The common algorithms that enable spectrum sensing are energy detection, matched filtering, cyclostationary detection, covariance based detector and wavelet-based sensing method. A matched filter is obtained by correlating a known signal with unknown signal to detect the presence of the template in the unknown signal. But the matched filter based detection requires the entire knowledge of the primary signals, which is not feasible for practical applications. In energy detection method, the energy of input signal is compared with some threshold energy value. The signal is said to be present at a specified frequency if the energy of the signal is beyond the energy level of the threshold. But, the execution of energy detection extremely degrades in presence of noise and interference power uncertainty and the detector fails to differentiate primary signal 
International Journal of Trend in Scientific Research and Development (IJTSRD) ISSN: 2456-6470

from the harmful interference. Cyclostationarity feature detection is a method for detecting primary user transmissions by utilizing the cyclostationarity features of the received signals but it doesn't make complete use of the characteristics of the modulated signals. In this paper, we proposed a spectrum sensing for digitally modulated primary signals using Bayesian detector (BD) to the utilization of spectrum, without the prior knowledge of the transmitted sequence of the primary signals. This method makes use of the prior observations of PU activity as well as the signaling information of the PU such as modulation order and symbol rate to improve the SU throughput and the overall spectrum utilization of both PUs and SUs.

\section{System Design}

In Spectrum sensing there are two hypotheses: the hypothesis that the PU is not present is $\mathscr{H} 0$ and the hypothesis that the PU is available or present is $\mathscr{H} 1$. In spectrum sensing, there are two important design parameters: the probability of SU to correctly detects the presence of primary signals known as probability of detection $(P D)$, and the probability of SU to falsely detects primary signals when PU is actually not present known as probability of false alarm $(P F)$. So we determine spectrum utilization as

$$
P(H 0)(1-P F)+P(H 1) P D
$$

and normalized SU throughput1 as

$$
P(H 0)(1-P F)
$$

Note that $P(\mathscr{H} 1) P D$ is PU throughput when there are active primary signals and the SUs finds the presence of these primary signals.

In order to find whether the available spectrum is being used by the primary user or not, the detection statistic $T D$ is being compared with a predetermined threshold $\epsilon$.

Probability of false alarm $P F$ is the probability of SU that chooses $\mathscr{H} 1$ while actually it is in fact $\mathscr{H} 0$ :

$$
P F=P(T D>\epsilon \mid H 0)
$$

Probability of detection PD is the probability of SU that correctly decides $\mathscr{H} 0$ when it is $\mathscr{H} 1$ :

$$
P F=P(T D>\epsilon \mid H 1)
$$

\section{Signal model}

Through the signal model, we have considered timeslotted primary signals in which $N$ primary signal samples are used to analyze the existence of PU signals. Similarly the PU symbol duration is $T$ which is known to the SU and at the secondary receiver, the received signal $r(t)$ is sampled at a rate of $1 / T$. For MPSK signals, the received signal of $k$-th symbol at the CR detector, $r(k)$

$r(k)= \begin{cases}n(k) & H_{0} \\ h e^{j \varphi n(k)}+n(k) & H_{1}\end{cases}$

Where $n(k)=n_{c}(k)+j n_{s}(k)$ is a complex AWGN signal with variance $N 0 \quad n_{c}(k)$ and $n_{s}(k)$ are respectively the real and imaginary part of $\mathrm{n}(\mathrm{k}), \varphi \mathrm{n}(\mathrm{k})=2 \pi \mathrm{n} / \mathrm{M}, \mathrm{n}=0,1, \ldots . . \mathrm{M}-1$ with equiprobability, and the propagation channel $\mathrm{h}$ is assumed to be constant within the sensing period. Let

$\boldsymbol{r}=\left[\begin{array}{lllll}r(0) & r(1) & \ldots \ldots & r(N-1)\end{array}\right]$ such that the SU receiver has no information with regards to the transmitted signals by the $\mathrm{PU}$ and $\varphi \mathrm{n}(\mathrm{k}), \mathrm{k}=$ $0,1, \ldots \ldots \mathrm{N}-1$ independent and identically distributed (i.i.d.) and independent of the Gaussian noise.

Detection statistics of energy detector (ED) is defined as the average energy of detected samples as

$$
T_{E D}=\frac{1}{N} \sum_{k=1}^{N}|r(k)|^{2}
$$

As the energy detector does not require the prior knowledge of the symbol rate, so we assume that the sample rate and symbol rate are similar. It is known that the optimal detector based on Bayesian rule or Neyman-Pearson theorem for binary hypothesis testing is to calculate the likelihood ratio and then make its choice by comparing the ratio with the threshold $\epsilon$. The likelihood ratio test (LRT) of the values $\mathscr{H} 1$ and $\mathscr{H} 0$ can be defined as

$T_{L R T}(r)=\frac{P\left(r \mid H_{1}\right)}{P\left(r \mid H_{0}\right)}$

\section{Optimal Detector Structure}

The probability density function (PDF) of received signals over $N$ symbol duration for hypothesis of $H 0$ is indicated as $\mathrm{p}(\mathbf{r} \mid \mathrm{H} 0)$, and can be written as

$$
p\left(r \mid H_{0}\right)=\prod_{k=0}^{N-1} \frac{e^{-|r(k)|^{2} / N_{0}}}{\pi N_{0}}
$$


International Journal of Trend in Scientific Research and Development (IJTSRD) ISSN: 2456-6470

Since the noise signals $\mathrm{n}(\mathrm{k}), \mathrm{k}=0,1 \ldots \ldots \mathrm{N}-1$ are i. Approximation in the Low SNR regime independent.

The PDF of received signals over $N$ symbol duration under hypothesis $H 1$ is denoted as $p(\boldsymbol{r} \mid H 1)$. With equiprobability of $\varphi n(k)=2 n \pi / M, n=0,1, \ldots, M-$ 1 , can be obtain as follows:

$p\left(r \mid H_{1}\right)=\prod_{k=0}^{N-1} \sum_{\varphi_{n}(k)} p\left(r(k) \mid H_{1}, \varphi_{n}(k) p \varphi_{n}(k)\right.$

Hence, the log-likelihood ratio (LLR), ln TLRT(r), is

$\sum_{k=0}^{N-1}\left(\operatorname{In}\left(\sum_{n=0}^{M-1} e^{\frac{2}{N_{0}} R\left[r(k) h^{*} e^{-j \varphi n(k)}\right]}\right)-\gamma-\operatorname{In} \mathrm{M}\right)$

Where $\gamma$ is the SNR of the received signal sample i.e $\gamma=\frac{|h|^{2}}{N_{0}}$

Let

$V_{n}(k)=\frac{2}{N_{0}} R\left[r(k) h^{*} e^{-j \varphi n(k)}\right]$

It is easy to derive the structure of the optimal detector (BD) for MPSK signals as:

$\mathrm{T}_{B D}=\frac{1}{N} \sum_{k=0}^{N-!} \operatorname{In}\left(\sum_{n=0}^{M / 2^{-1}} \cosh \left(v_{n}(k)\right)\right)_{<}^{>} \gamma+$

$\operatorname{In} \frac{M}{2}+\frac{\operatorname{In} \epsilon}{N}$

Even though the detector is optimal, it is very complicated to use in practice. So we will simplify the detector when the SNR is very low or very high as follows.

\section{Bayesian detector (ABD) structure through the Approximations in the Low and High SNR regimes}

In this paper we have given the theoretical analysis (detection performance and threshold) in low SNR regime for the suboptimal detector to analyze complex MPSK signals $(M=2$ and $M>2)$ and compare with the results for real BPSK primary signals [4].
We study the approximation of our proposed detector in the low SNR regime for the complex MPSK modulated primary signals. When $x \rightarrow 0, \cosh (\mathrm{x}) \approx$ $1+\frac{x^{2}}{2}$ and $(1+x) \approx x$ we can obtain

$$
\sum_{n=0}^{N-1} \operatorname{In}\left(\sum_{n=0}^{\frac{M}{2}-1} \cosh \left(v_{n}(k)\right)\right)
$$

Through approximation, the detector structure becomes:

$$
T_{L-A B D}=\frac{1}{N} \sum_{k=0}^{N-1}|r(k)|^{2}>\left(\gamma+\frac{I n \epsilon}{N}\right)
$$

\section{ii. Approximation in the High SNR regime}

In this section we consider the high SNR regime. When $x>>0, \cosh (\mathrm{x}) \approx \frac{e^{x}}{2}$ or when $\mathrm{x}<<0, \cosh (\mathrm{x}) \approx$ $\frac{e^{-x}}{2}$

The detector structure becomes

$\mathrm{T}_{\mathrm{H}-\mathrm{ABD}}=$
$\sum_{\mathrm{k}=0}^{\mathrm{N}-1} \operatorname{In}\left(\sum_{\mathrm{n}=0}^{\mathrm{M} / 2-1} \mathrm{e}^{\frac{2}{\mathrm{~N}_{0}} \mathrm{R}\left[\mathrm{r}(\mathrm{k}) \mathrm{h}^{*} \mathrm{e}^{-\mathrm{j} \varphi_{\mathrm{n}}(\mathrm{k})}\right]}\right) \underset{<}{>} \gamma+\operatorname{InM}$

In such a special case of MPSK signals, we consider a real signal model for the BPSK modulated primary signals. In the high SNR regime, the suboptimal Bayesian detector employs the addition of received signal magnitudes to detect the presence of primary signals which indicates that in this regime energy detector is not optimal.

\section{RESULTS}

We plot a result for Secondary users' throughput of H-ABD vs. SNR (dB) for BPSK signal at high SNR in fig. 1 it is observed that as SNR increases the secondary user's throughput also increases. 


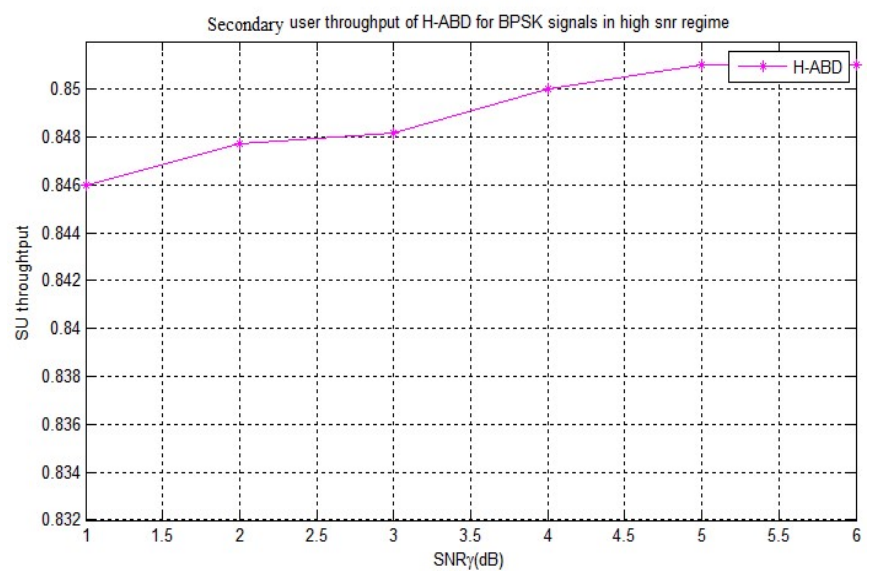

Fig. 1: Secondary users throughput of SNR vs. HABD for BPSK signal at H SNR

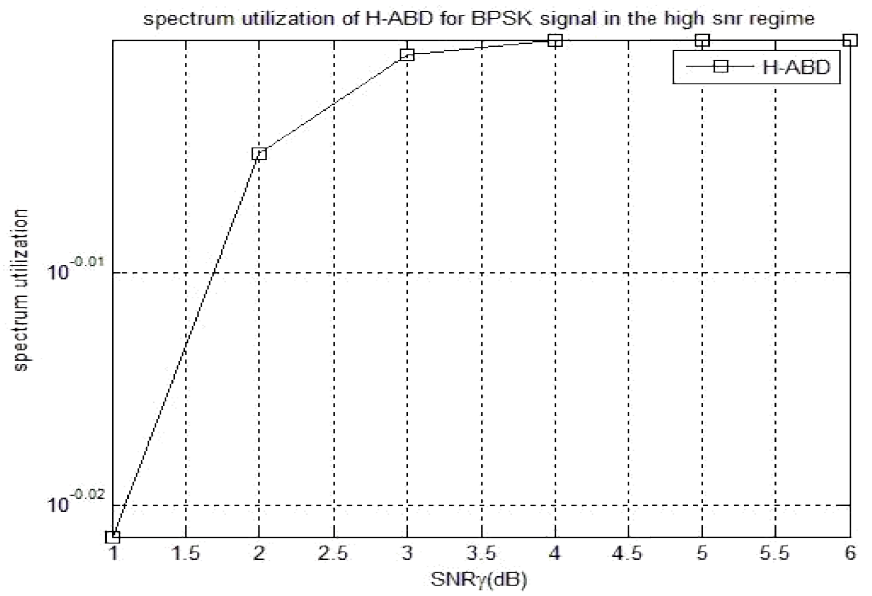

Fig. 2: Spectrum utilization of SNR (dB) vs. H-ABD for BPSK signal at high SNR.

In the simulation for BPSK signals it is shown that both BD and H-ABD has higher Pd and lower Pf than ED. so that $\mathrm{BD} / \mathrm{H}-\mathrm{ABD}$ has a better performance in terms of secondary users' throughput and spectrum utilization. Also it is shown fig. 2 that as SNR increases the spectrum utilization of H-ABD also increases for Bpsk signals. The results confirm that energy detector is not optimal in high SNR regime.

\section{Conclusion}

In this paper, we surveyed on optimal Bayesian detector in order to detect known order of MPSK modulated primary signals over AWGN channels, which is based on Bayesian decision rule. Through approximations, we have found that at Low SNR regime the Bayesian detector has similar structure as energy detection. But at High SNR energy is the sum of signal magnitude, where the Bayesian detector performs better in terms of spectrum utilization as well as secondary user's throughput than energy detector. And hence this improves the detection probability for given false alarm probability.

\section{References}

1. Shoukang Zheng, Pooi-Yuen Kam, Ying-chang Liang, and Yonghong Zheng, "Spectrum sensing for digital primary signals in cognitive radio: A Bayesian approach for maximizing spectrum utilization," IEEE transactions on wireless communications, vol. 12, no. 4, April 2013, pp. 1774-1782.

2. T. Y"ucek and H. Arslan, "A survey of spectrum sensing algorithms for cognitive radio applications," IEEE Communication Surveys \& Tutorials, vol. 11, no. 1, 2009 pp. 116-130.

3. Miguel L'opez-Ben'itez and Fernando Casadevall, "Signal Uncertainty in Spectrum Sensing for Cognitive Radio", IEEE transaction Communication, vol. 61, no. 4, April 2013, pp. 1231-1241.

4. H. Urkowitz, "Energy detection of unknown deterministic signals," Proc. IEEE, vol. 55, no. 4, 1967, pp. 523-531.

5. Jun Fang, Ning Han, Hongbin Li, \Multiantenna assisted spectrum sensing for cognitive radio," International Journal of Engineering and Innovative Technology, vol.1, issue 2, May 2010, pp.11-15.

6. Fadel F. Digham, Alouini, Marvin K. Simon, "On the Energy Detection of Un-known Signals over Fading Channels," IEEE communications surveys \& tutorials, vol.11, no.1, Jan 2007, pp.116-130.

7. Y. H. Zeng and Y.-C. Liang, "Spectrumsensing algorithms for cognitive radio based on statistical covariances," IEEE Transaction vehicular technology, vol. 58, no. 4, 2009, pp. 1804-1815.

8. Ying-Chang Liang and Rui Zhang, "Blindly Combined Energy Detection for Spectrum Sensing in Cognitive Radio," IEEE transactions on vehicular technology, vol.59, no.4, May 2008, pp.1877-1886.

9. Shoukang Zheng,Pooi-Yuen Kam,Ying-Chang Liang,and Yonghong Zeng,"Spectrum Sensing for Digital Primary Signals in Cognitive Radio: A Bayesian Approach for Maximizing Spectrum Utilization", IEEE transaction communication, vol. 12, no. 4, April 2013, pp. 1774-1782.

10. Y. -C. Liang, Y. H. Zeng, E. Peh, and A. T. 
International Journal of Trend in Scientific Research and Development (IJTSRD) ISSN: 2456-6470

Hoang, "Sensing-throughput tradeoff for cognitive radio networks," IEEE transaction wireless communication, vol. 7, no. 4, Apr. 2008, pp. 1326-1337.

11. Y. H. Zeng, Y.-C. Liang, A. T. Hoang, and R. Zhang, "A review on spectrum sensing for cognitive radio: challenges and solutions," EURASIP J. Advances in Signal Process., no. 1, 2010, pp. 1-15.

12. S. Zheng, P.-Y. Kam, Y.-C. Liang, and Y. Zeng, "Bayesian spectrum sensing for digitally modulated primary signals in cognitive radio," in Proc. 2011 IEEE vehicular technology Conf. - Spring, pp. 1-5. 